\title{
SCN lesions abolish ultradian and circadian components of activity rhythms in LEW/Ztm rats
}

\author{
FRANZISKA WOLLNIK AND FRED W. TUREK \\ Department of Neurobiology and Physiology, Northwestern University, Evanston, Illinois 60208
}

\begin{abstract}
Wollnik, Franziska, AND Fred W. TUREK. SCN lesions abolish ultradian and circadian components of activity rhythms in LEW/Ztm rats. Am. J. Physiol. 256 (Regulatory Integrative Comp. Physiol. 25): R1027-R1039, 1989.-A trimodal locomotor activity pattern has been observed in LEW/Ztm rats. Complete and partial lesions of the suprachiasmatic nucleus (SCN) were used to determine whether the same neural substrate may underlie the circadian rhythms and the ultradian modulation of wheel-running activity in these rats. Whereas sham lesions had little or no effect on the wheel-running activity pattern, complete SCN lesions resulted in a complete loss of circadian and ultradian activity components under free-running or 12:12 h light-dark cycle (12:12 LD) conditions. Ultradian and circadian activity components were still present after partial SCN lesions. Periodogram analysis for any given animal revealed that the ultradian periods were always submultiples of the entrained or free-running circadian period. Furthermore there was a high correlation between the amplitudes of circadian and ultradian spectral estimates, but with a different slope in males and females. These results indicate that in LEW/Ztm rats the $\mathrm{SCN}$ contributes to the control of both the circadian wheelrunning rhythm and the trimodal ultradian modulation of that behavior.
\end{abstract}

circadian rhythms; ultradian rhythms; multiple circadian oscillators; coupling; splitting

EXPERIMENTAL RESULTS OBTAINED over the past 15 years indicate that circadian rhythms in mammals are regulated by an endogenous multioscillatory pacemaker system located in or close to the suprachiasmatic nuclei (SCN) in the hypothalamus (for reviews see Refs. 20, 21, 24). Temporal variations with shorter periods, called ultradian or short-term rhythms, are known to exist in the behavioral patterns of small mammals. However, unlike circadian rhythms, these ultradian behavioral rhythms have no obvious counterpart in the environment, and their origin and function remain unclear (3, $8,10)$.

In laboratory rats, tri- or multimodal activity patterns have been described for intact animals $(2,23)$, but they are much more pronounced during the early developmental stage (15) and under prolonged exposure to constant illumination $(1,12-14)$. A study of locomotor activity patterns in different inbred strains of laboratory rats (6) has shown that the inbred strain LEW/Ztm exhibits a very precise and reproducible trimodal activity pattern in locomotor activity as well as in body temperature and heart rate (5). This pattern generates ultradian peaks in the range of 4 and $4.8 \mathrm{~h}$ when tested by different methods of period analysis (i.e., $\chi^{2}$ periodogram and harmonic spectral analysis). It has been shown recently that the trimodal activity pattern of this strain exhibits substantial heritability with a general recessive mode of inheritance (29). The expression of a trimodal activity pattern also depends on the hormonal status of the adult animals. Ultradian modulation of the activity pattern is inhibited in both male and female LEW/Ztm rats by serum levels of estrogen that correspond to those observed on the day of proestrus and estrus (28).

The question therefore arises as to whether the trimodal activity pattern of the LEW/Ztm strain is a manifestation of a unique phase coupling of multiple circadian oscillators or whether the circadian system modulates the expression of one or several ultradian oscillators that are anatomically and functionally distinct from the circadian system. An independent ultradian rhythm that is gated by the circadian system has been shown to generate the feeding and locomotor activity patterns in the common vole, Microtus arvalis (8). In this species a total lesion of the SCN did not result in the loss of ultradian rhythmicity in wheel running and feeding, whereas the circadian pattern was abolished (10). These results indicate the existence of an autonomous ultradian oscillator outside the SCN in the common vole.

The purpose of the present study was to examine the effect of SCN lesions on the trimodal activity pattern of male and female LEW/Ztm rats maintained in running wheels under both free-running and entrained 12:12 h light-dark cycle (12:12 LD) conditions. The results of the present study demonstrate that ultradian activity components do not persist after complete SCN lesions, thereby indicating that the trimodal activity pattern of $\mathrm{LEW} / \mathrm{Ztm}$ rats is fundamentally different from the ultradian rhythms observed in feeding and wheel-running behavior in voles.

\section{METHODS}

Animals and housing. Rats of the inbred strain LEW/ Ztm, originally obtained from the central animal laboratory at the Hannover Medical School (FRG), were bred and raised in our laboratory under controlled environmental conditions (12:12 light-dark cycle, lights on at 0600 , room temperature $22 \pm 1^{\circ} \mathrm{C}$ ). Of 19 male and 16 female rats entering the study, half of the animals were blinded under anesthesia $(100 \mathrm{mg} / \mathrm{kg}$ ketamine hydrochloride and $10 \mathrm{mg}$ xylazine im) by bilateral orbital enucleation at 70 days of age. Blinded and sighted ani- 
mals were placed in individual cages containing an activity wheel and a small adjacent housing compartment on day 1 of the experiment. The running wheels were arranged on racks with three different levels, and the animals of different experimental groups were assigned to the wheels in a random fashion. Commercial rat chow and water were available ad libitum. Wheel-running activity was recorded for a total of 90 days.

Lesions and histological analysis. Seventeen male (8 sighted, 9 blinded) and 14 female (7 sighted, 7 blinded) $\mathrm{LEW} / \mathrm{Ztm}$ rats received bilateral SCN lesions between days 35 and 50 of the experiment. All lesions were made during the inactive period of the activity rhythm of both sighted and blinded animals under deep anesthesia (same as for blinding). Electrolytic lesions in the region of the $\mathrm{SCN}$ were made with tungsten wire electrodes $(0.2-\mathrm{mm}$ diam) coated with Epoxylite except for the cross section of the tip. The stereotaxic placement of the lesion electrode was accomplished with a stereotaxic instrument. With the incisor bar $5.0 \mathrm{~mm}$ above the interaural line, the coordinates were $2.1 \mathrm{~mm}$ anterior to bregma and \pm 0.3 lateral and $8.4 \mathrm{~mm}$ below dura. A 2-mA direct current was passed for $15 \mathrm{~s}$ on each side. Four control animals received sham operations during which they were anesthetized, placed in the stereotaxic apparatus, and manipulated in the same way as the SCN-lesioned animals, except that the electrode was lowered to a point just dorsal to the SCN, and no current was applied. Twentyfour hours after surgery, the animals were transferred back into the wheels.

When activity recording was terminated, the animals were anesthetized (same as for blinding) and perfused with saline followed by $10 \%$ Formalin. Their brains were removed and stored in 10\% Formalin, 30\% (wt/vol) sucrose. Frozen $60-\mu \mathrm{m}$ coronal sections were cut and stained with thionine. Analysis of the lesions consisted of two procedures, a qualitative assessment of structures impinged on by the lesion, and a quantitative determination of the volume of remaining SCN tissue. SCN volumes of the four brains with sham lesions were calculated from the slice thickness and area of the SCN by projecting each section of the SCN onto paper (magnified $\sim \times 45$ ) and drawing the borders of the nucleus. The volume of remaining SCN tissue in lesioned rats was measured by comparing each section with the corresponding section of a sham-lesioned brain and is expressed as a percentage of the average volume of shamtreated rats.

Data collection and analysis. Wheel-running activity was monitored on line with a microcomputer (Apple II+) and was recorded in terms of wheel revolutions per 5min interval. Data were stored on floppy disks every 24 h. For visual inspection, the activity records were "double plotted," i.e., each horizontal line of the record represents two consecutive 24 -h periods, and the record for each day is plotted below the record of the preceding day. Total counts of wheel revolutions for each 30 -min interval are shown as a histogram, with the height of the histogram being proportional to the number of counts. Maximum height of the histogram represents $>300$ counts [i.e., an average of 10 counts/min (cpm)], whereas a blank space indicates that there were $<30$ counts (i.e., an average of $1 \mathrm{cpm}$ ).
A $\chi^{2}$ periodogram (22) and a "harmonic spectral analysis" $(11,19)$ were used to test for the presence of circadian and ultradian rhythms. The two different approaches have been developed from completely different statistical models, and results of both methods were always compared to check the reliability of each method. Both techniques and their application to circadian and ultradian data have been described in greater detail elsewhere (28), including a test with artificial data of different periods (ultradian and/or circadian), amplitude, and waveforms (sinecosine, square wave, saw tooth) in the presence of different levels of noise.

The blocks of data subjected to both analyses were two intervals of 21 days each of base-line data beginning 35 days before surgery (days 15-35 or 29-49) and two intervals of 21 days each of postsurgical data beginning 7-10 days after surgery (days $57-77$ or 71-91). The data were analyzed in 5- and 20-min intervals in the periodogram and the power spectrum, respectively. Power spectra of individual animals with the same experimental protocol were pooled. Differences in the amplitude of certain spectral estimates before and after SCN lesion were tested with a two-way analysis of variance (ANOVA) and Scheffé $t$ test after logarithmical transformation of the spectral values. Periodogram and power spectrum analyses were also performed on 2- and 7-day intervals of data to examine the possibility that ultradian rhythms with variable periods are present in the data set. These analyses showed that 2-day intervals are too short for the detection of any kind of rhythmicity. The results obtained for a set of three 7-day intervals differed from those obtained for one 21-day interval only in that the probability for the detection of weak rhythmic components was much higher in the longer time interval. Periodogram and power spectrum analyses over 21 days are therefore more convincing methods with which to check the arrhythmic state of animals.

\section{RESULTS}

Data were obtained from 35 animals, 4 sham lesions, 17 complete SCN lesions, and 14 partial or missed SCN lesions. Although the results from all animals were used in the data analysis, presentation of the data on an individual basis is restricted to 12 representative animals, 4 with complete lesions, 6 with partial or missed SCN lesions, and 2 controls.

\section{Histology}

Complete SCN lesions. Figure 1 shows the extent of the lesion in the four representative animals with complete lesions of the SCN from which data appear in the other figures. Animals $L E W \delta 33$, $\$ 35$, $\$ 25$, and $\$ 16$ had complete SCN lesions. $L E W \$ 33$ had a fairly large, complete lesion that extended $\sim 1.0 \mathrm{~mm}$ caudal and $\sim 1.7 \mathrm{~mm}$ dorsal to the SCN. Most of the medial preoptic area and anterior hypothalamus as well as parts of the paraventricular, ventromedial, and arcuate nuclei were destroyed. $L E W \delta 33$ was the only animal in which the lesion extended to the region of the arcuate nuclei. Rats $L E W \delta 35$, $\$ 25$, and $\$ 16$ had small lesions with little 


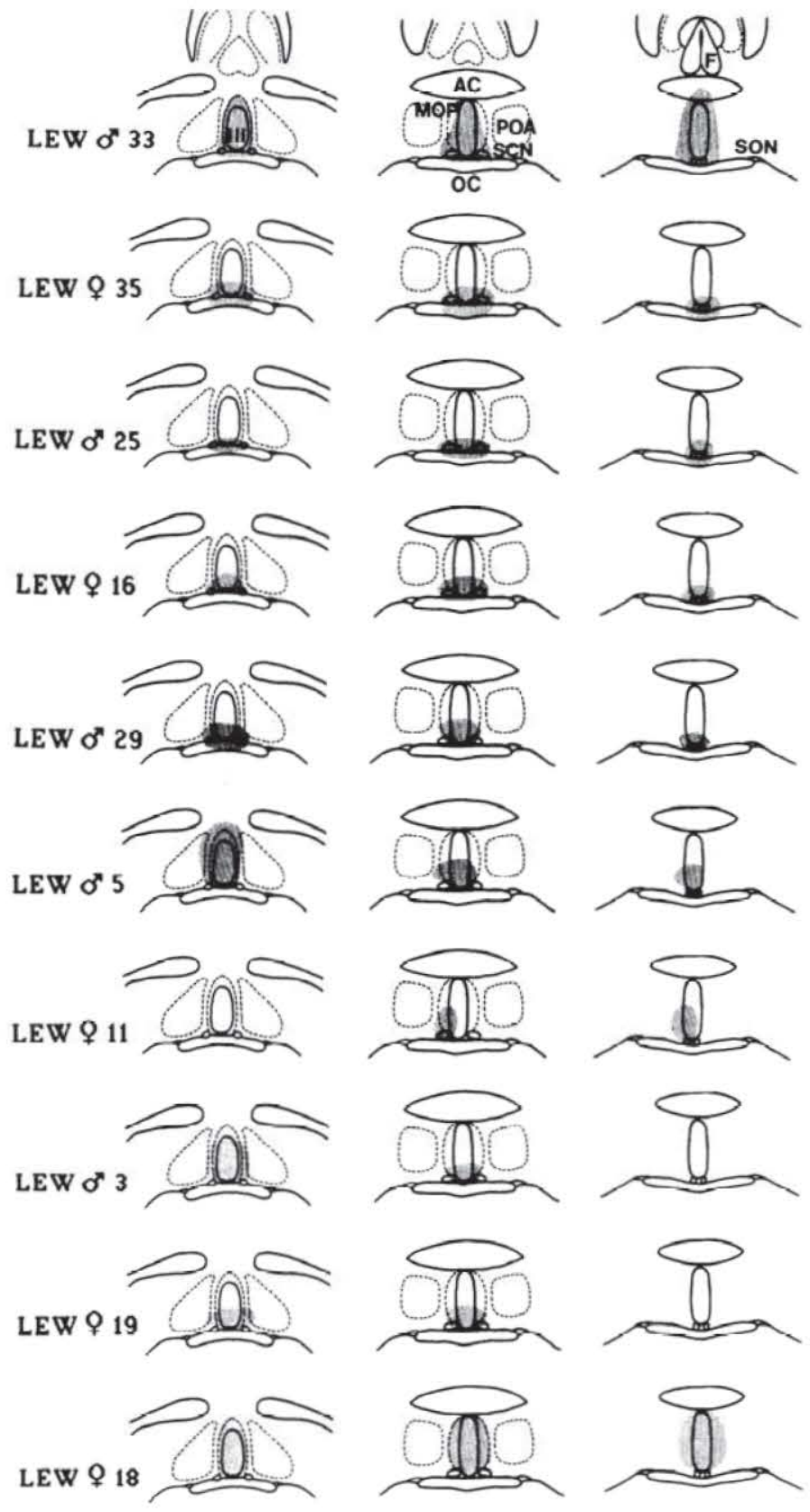

FIG. 1. Extent of lesion (gray area) in 10 representative animals at 3 levels of SCN. Each column represents 1 level of SCN and sections are $\sim 200 \mu \mathrm{m}$ apart from rostral (left) to caudal (right). Diagrams are composites drawn from brains of sham-lesioned rats of comparable age. Four animals with complete lesions (LEW $333, \$ 35, \delta 25, \$ 16)$ and 6 animals with partial lesions are shown. AC, anterior commissura; F, fornix; MOP, medial preoptic area; OC, optic chiasm; POA, peripheral preoptic area; $\mathrm{SCN}$, suprachiasmatic nucleus; $\mathrm{SON}$, supraoptic nucleus; III, third ventricle.

damage to the surrounding area, except for the optic chiasm in LEW 35 and $\$ 25$.

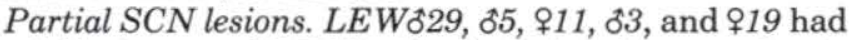
partial lesions, and $L E W$ 웅 18 had a lesion that completely missed the SCN. LEWð̋29 had a lesion that destroyed $\sim 85 \%$ of the SCN. The lesion was small and damaged little of the surrounding area, except for a portion of the chiasm ventral to the rostral part of the SCN. LEW $\$ 5$ had a large lesion, but the main part of the lesion was anterior to the $\mathrm{SCN}$, and the lateral and caudal parts of the SCN were intact. The lesion destroyed $\sim 50 \%$ of the
$\mathrm{SCN}$ and also damaged the medial portion of the preoptic area and parts of the anterior hypothalamus. In rat $L E W+11, \sim 30 \%$ of the SCN was destroyed. One SCN was completely spared, and $\sim 60 \%$ of the other SCN was destroyed with damage mainly in the medial dorsal portion. $L E W \$ 3$ and $\$ 19$ had lesions that destroyed $\sim 15-$ $20 \%$ of the SCN. The damage to the SCN was mainly in the medial and rostral dorsal portions. The caudal parts of the SCN were spared. For $L E W \$ 18$, the lesion spared the $\mathrm{SCN}$, falling directly caudal to these nuclei. The lesion extended dorsally into the anterior hypothalamus and caudally to the anterior ventromedial hypothalamus. The lesion did not damage the arcuate nuclei.

\section{Wheel-Running Activity Pattern}

After surgery, the mean daily activity levels were reduced to an average of $85 \%$ of base line in the shamlesioned rats and to an average of 28 and $12.5 \%$ of base line in the partial and complete lesioned animals, respectively. This reduction in mean activity was small but statistically significant for the sham-lesioned animals $(P$ $<0.05)$ and significant $(P<0.01)$ for the partial and complete lesioned animals and was maintained for at least 50 days after surgery.

Intact animals. The wheel-running activity of the LEW/Ztm rats before surgery was characterized by a trimodal pattern, which confirms previous results for the overall activity patterns of this inbred strain $(6,28)$. The sham-lesioned animals $L E W+21$ and $\$ 30$ illustrate the wheel-running activity pattern typically seen in LEW/ Ztm rats (Fig. 2). Periodogram analysis of these records and of all experimental animals before surgery detected significant circadian and ultradian components. However, an ultradian period of, for example, $4.8 \mathrm{~h}$ may also produce periodogram peaks at $9.6,14.4$, and $19.2 \mathrm{~h}$, thereby generating a pattern of repeating peaks. Therefore only the smallest submultiple of a sequence of repeating peaks represents the "true" period of an ultradian rhythm. In the sighted animals $(n=16)$, the ultradian components had periods of $4,4.8$, or $6 \mathrm{~h}$ under $12: 12 \mathrm{LD}$ entrainment, whereas in the free-running blinded animals $(n=19)$ the ultradian components had similar periods (e.g., 4.07, 4.92, or $6.17 \mathrm{~h}$ ). A comparison of ultradian and circadian periods in the periodograms of sham-lesioned and intact animals showed that the periods of ultradian peaks were always submultiples of the observed circadian period (See Relationship between ultradian and circadian rhythms). Ultradian components were more prominent in male animals, because the trimodal activity pattern of the females was interrupted every $4-5$ days by the day of estrus (e.g., $L E W$ Q 16 in Fig. $4 A$ ). This estrus-correlated modulation of the activity pattern in females is described in detail in Ref. 30.

Harmonic spectral analysis confirmed the findings obtained by periodogram analysis. Figure $3 \mathrm{~A}$ shows pooled power spectra of the sham-lesioned animals for four different time intervals. Because of the limited resolution of the spectral analysis, no differences were detected between free-running and 12:12 LD-entrained animals. Therefore the results for the different light schedules were combined. However, results are presented separately for males and females because differences 
LEW 921
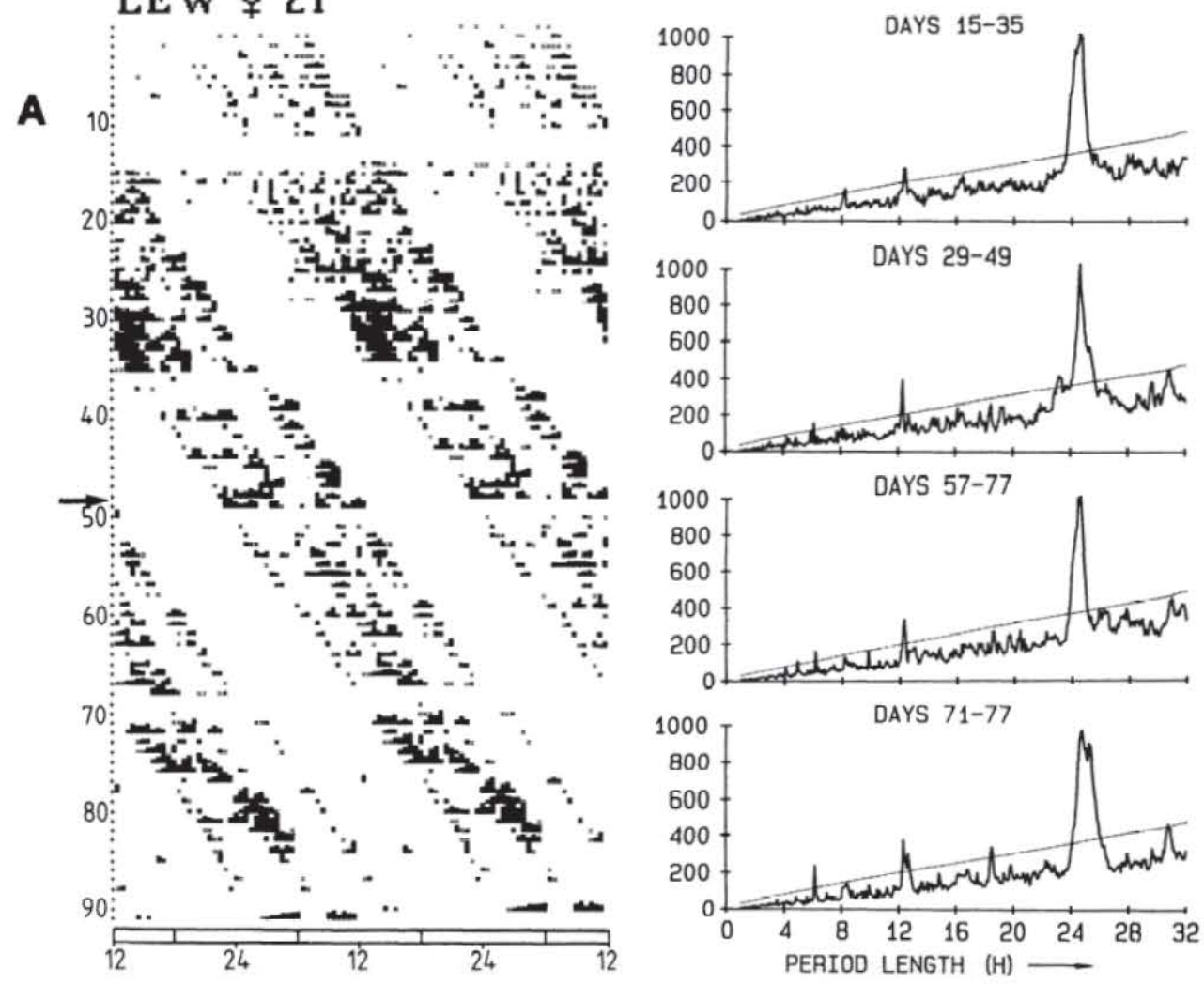

\section{LEW O' 30}

B

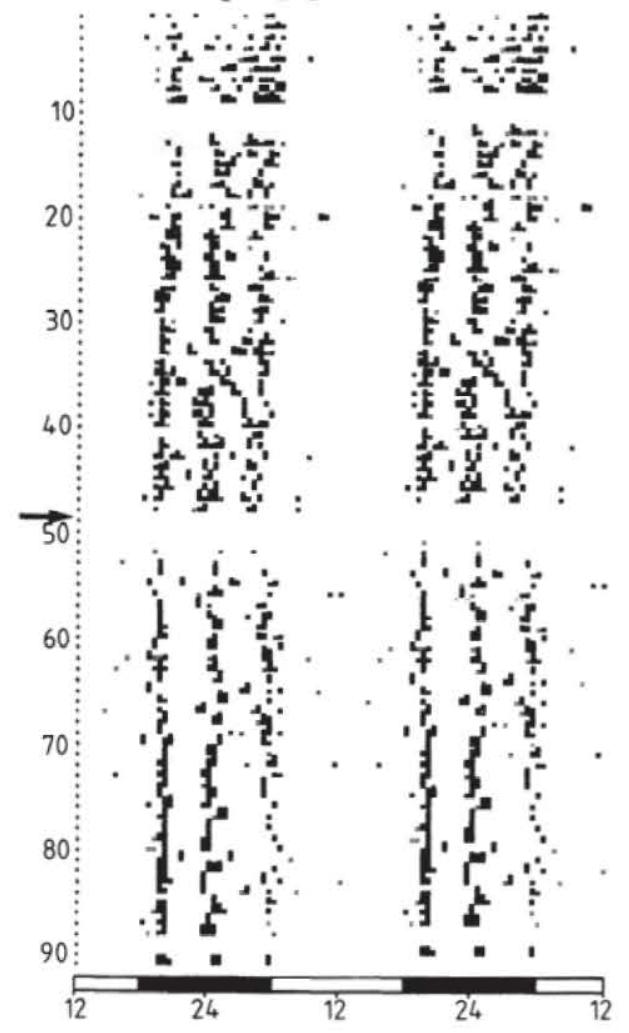

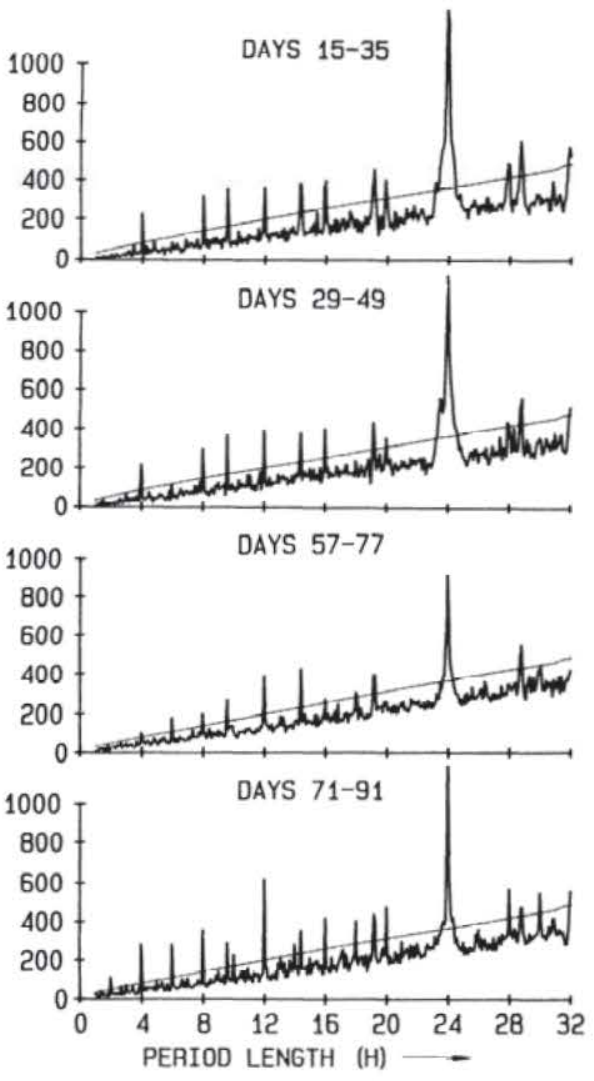

FIG. 2. Double-plotted wheel-running activity records of 2 sham-lesioned LEW/Ztm rats, LEW $\$ 21(A)$ and $\delta 30(B)$. In this and following records, day of sham or SCN lesion is marked with an arrow. For animals under 12:12 h light-dark cycle (12:12 LD) entrainment, light schedule is indicated by dark bars on time axis. Blank lines, days with data missing because of power failure or system errors. Each record is accompanied by 4 periodograms representing an interval of 21 days ( 2 intervals before, and 2 intervals after surgery). Sloping line in periodograms represents significance level, $P<0.01$, derived according to Ref. 22. 
A

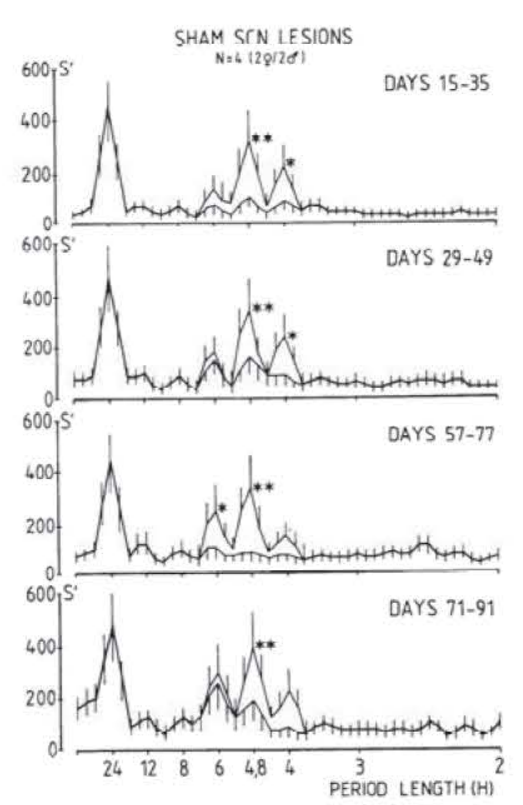

B

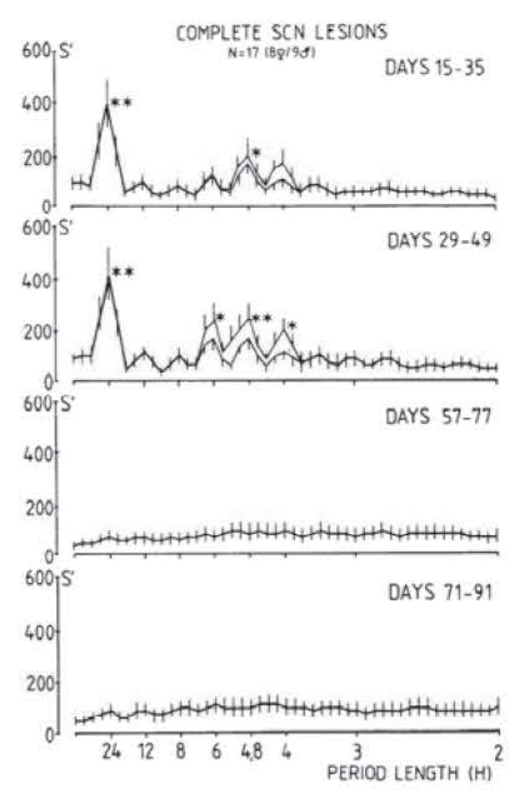

C

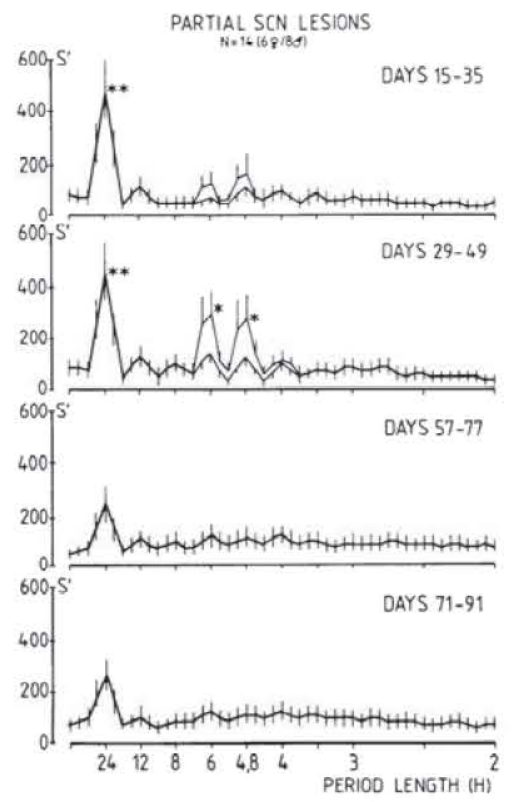

FIG. 3. Mean pooled power spectra for 4 different time intervals for all LEW/Ztm rats with sham SCN lesions $(A)$, complete SCN lesions $(B)$, and partial SCN lesions $(C)$. Vertical lines, $95 \%$ confidence limits of spectral estimates. Amplitude values of distinct spectral estimates were subject to a 2-way ANOVA and Scheffé multiple $t$ test. Data were analyzed with a $2 \times 4$ factorial experimental design ( 2 levels for males and females by 4 levels of analyzed time intervals). When spectra diverged for male and female rats, separate data are shown. Higher amplitudes in ultradian spectral values were always found in male animals. * Significant differences between ultradian spectral values obtained for male and female animals $(A)$ or significant differences between spectral estimates before and after surgery $(B, C)$ ( $\left.P<0.05,{ }^{* *} P<0.01\right)$.

were statistically significant $(P<0.05)$ in the amplitudes of the 4.8- and 4-h spectral estimates. Ultradian components with either 4-, 4.8-, or 6-h periods were detected in the sham-lesioned animals as well as in all other animals before surgery (Fig. 3, $B$ and $C$ ), with the amplitude of the 4- and 4.8-h spectral estimates being consistently higher in male animals.

Complete SCN lesions. Figure 4 shows the wheel-running activity of four representative animals in which the lesion totally destroyed the SCN. Visual inspection of the wheel-running activity records suggested a loss of rhythmicity after a complete SCN lesion in sighted as well as in blinded animals. Periodogram analysis of these activity records confirmed the loss of ultradian or circadian periodicities corresponding to the ultradian and circadian peaks seen before surgery. In some cases, very small but significant peaks were detected in the periodogram as, for example, a period of $13.0 \mathrm{~h}$ for $L E W \$ 16$, days 71-91 (Fig. 4A). The periods represented by these peaks were always longer than $7 \mathrm{~h}$ and showed no repeating pattern as did the ultradian peaks before surgery. Spectral analysis also confirmed the loss of circadian and ultradian components in the SCN-lesioned animals (Fig. $3 B$ ). Comparison of distinct spectral estimates revealed a statistically significant $(P<0.01)$ decrease in the amplitude of the 24-h spectral estimates after complete SCN lesions. The decrease in the amplitude for the 6-, 4.8-, and 4-h spectral values was significant $(P<0.05)$ only in the male animals.

Partial SCN lesions. Examples of activity records of animals with partial SCN lesions are shown in Fig. 5.
$L E W \$ 29$, with a nearly complete destruction of the SCN $(85 \%)$, showed a partial recovery of the activity pattern. First, a single activity bout appeared that was free running with a circadian period. After 20 days it was followed by a second component, generating a bimodal activity pattern. Periodogram analysis of the last 21 days (days 71-91) revealed an ultradian peak at $4.12 \mathrm{~h}$ and a circadian peak at $24.75 \mathrm{~h}$. LEW $\$ 5$, which had $50 \%$ destruction of the SCN, showed a weak free-running circadian rhythm without an obvious separation into three activity bouts after the surgery. Periodogram analysis of the data, however, detected a significant ultradian peak at $6.17 \mathrm{~h}$ for days 57-77 and days 71-91 and three peaks in the circadian range $(24.25,24.42$, and $24.67 \mathrm{~h})$ for the last 21 days of the record. Different circadian peaks, but no ultradian peaks, were detected in the record of $L E W+11$, which had a $30 \%$ lesion of the SCN. During the first 30 days after surgery, significant peaks were found at 24.08 and $24.75 \mathrm{~h}$. Periodogram analysis for the last 21 days of the record for $L E W+11$ (days 64-84) detected four separate circadian peaks $(24.33,24.67,24.83$, and $25.08 \mathrm{~h}$ ). The observation of multiple circadian peaks can be interpreted as a "dissociation of activity" into several components that follow a pattern and time course similar to one seen in the activity rhythms of a few individual rats (7). In $L E W \$ 3$ and $\$ 19$, which had lesions of only 15$20 \%$ of the SCN, the trimodal activity pattern was maintained, although the level of activity was obviously decreased. For $L E W \delta 3$, ultradian peaks actually became more prominent after surgery. Periodogram analysis revealed a circadian peak at $24.5 \mathrm{~h}$ and ultradian peaks at 

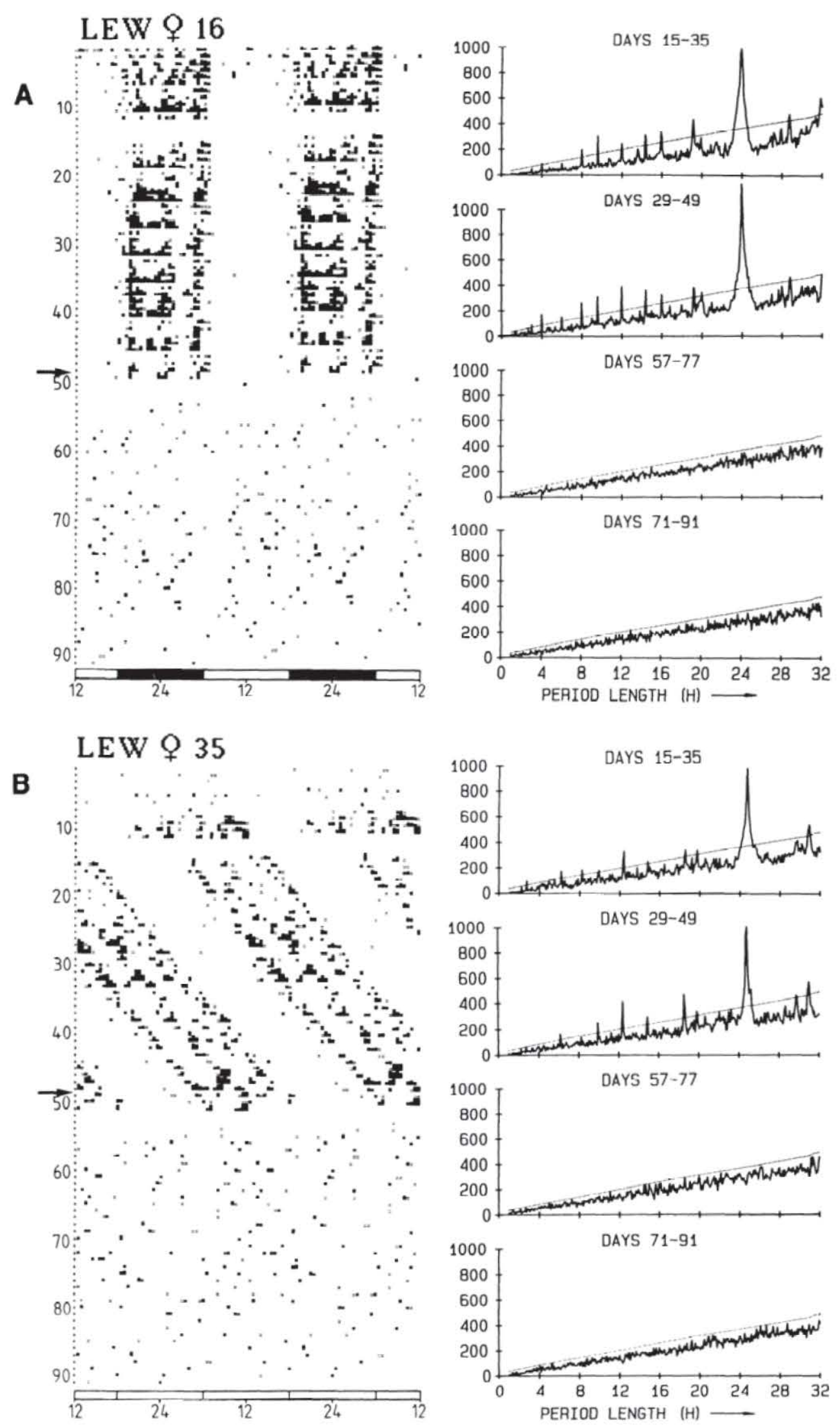

FIG. 4. Double-plotted wheel-running activity records of $4 \mathrm{LEW} / \mathrm{Ztm}$ rats with complete $\mathrm{SCN}$ lesions, $L E W$ \$16 $(A)$, \&35 $(B), \delta 25(C)$, and $\$ 33$ $(D)$. For further explanation see Fig.

4.08 and $6.08 \mathrm{~h}$, with the 4.08 -h component being more prominent during days 50-70 and the 6.08-h component during days 64-84. Periodogram analysis of $L E W \delta 19$ showed ultradian peaks at 4.92 and $6.17 \mathrm{~h}$ before surgery and ultradian peaks at 4.0 and $4.92 \mathrm{~h}$ after the surgery. It is noteworthy that this animal showed an ultradian peak at exactly $4.0 \mathrm{~h}$ and at all multiple periods, including $24.0 \mathrm{~h}$, despite the fact that it was blinded. The other 


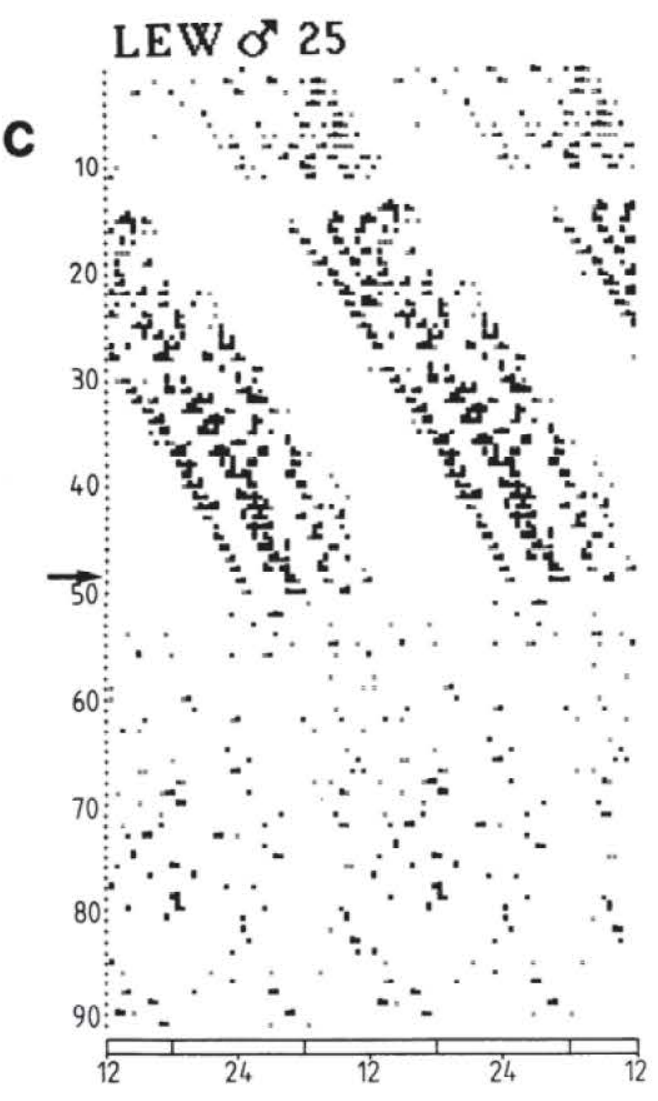

\section{LEW O' 33}

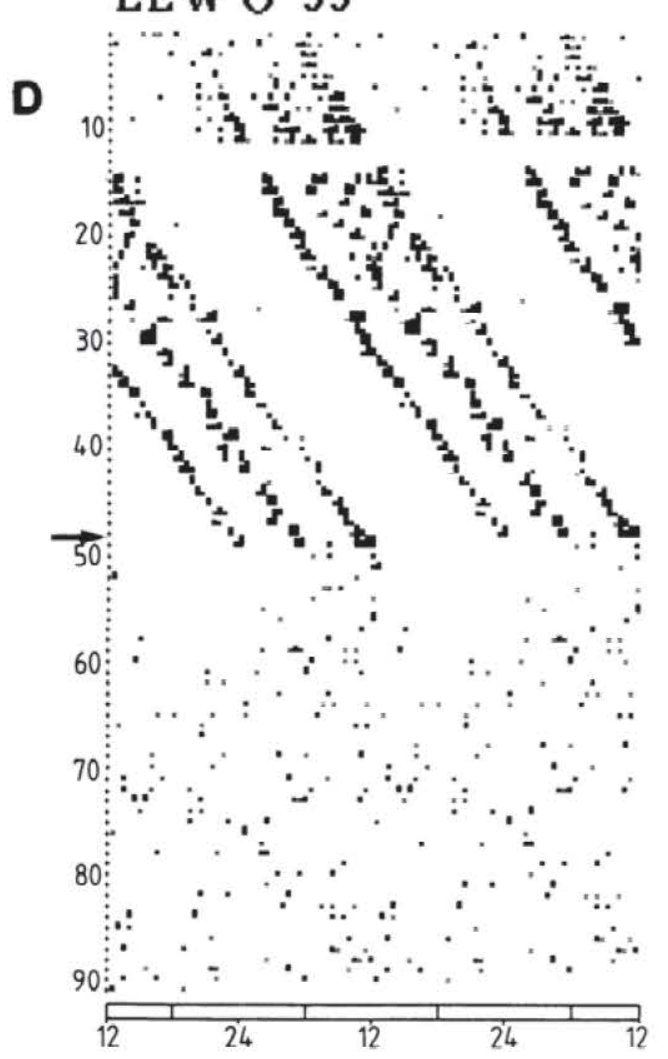

free-running ultradian component with a period of 4.92 $\mathrm{h}$ was a submultiple of the main circadian period of 24.58 h. The lesion in $L E W$ 이, which actually spared the SCN completely, resulted in a total loss of circadian and ultradian rhythmicity. The lesion was just caudal to the
DAYS $15-35$

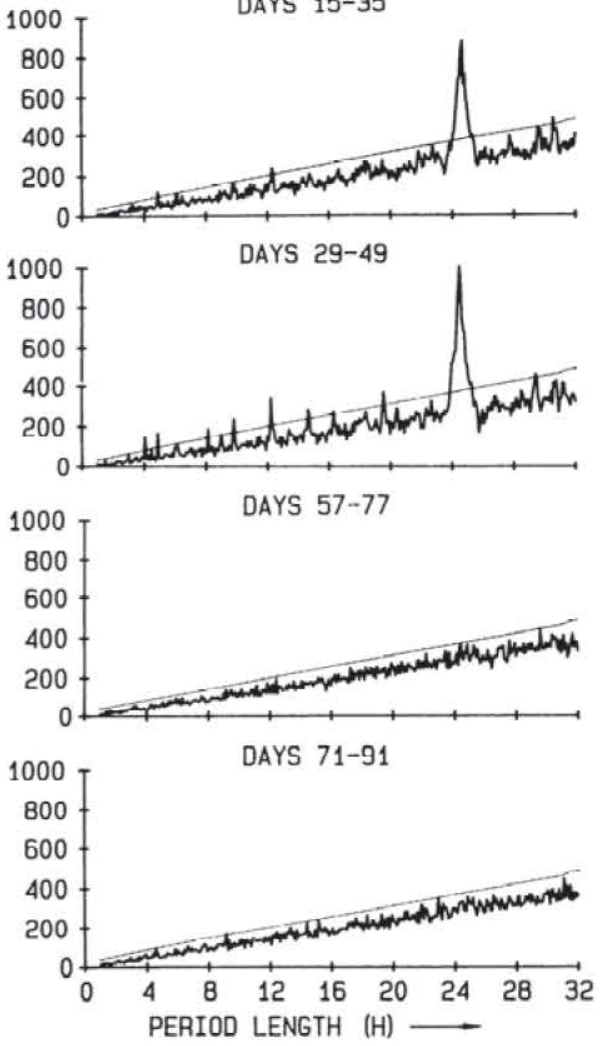

FIG. 4. Continued.
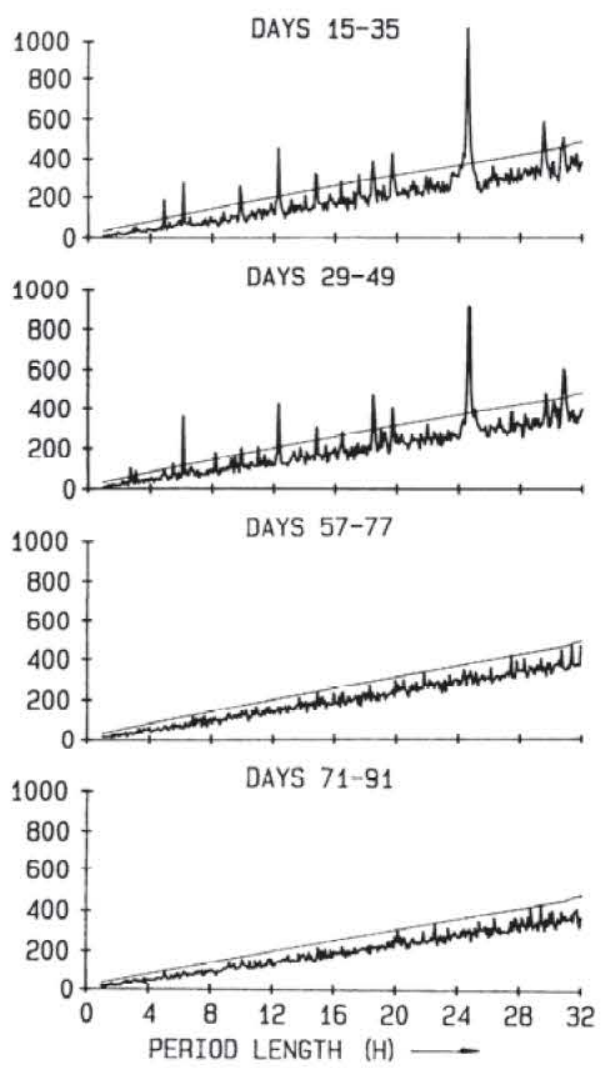

SCN and therefore isolated it from structures dorsal and caudal to the SCN.

Similar to the periodogram analysis, spectral analysis (Fig. $3 C$ ) confirmed that partial SCN lesions did not abolish circadian rhythmicity but did induce a significant 


\section{LEW Ơ 29}
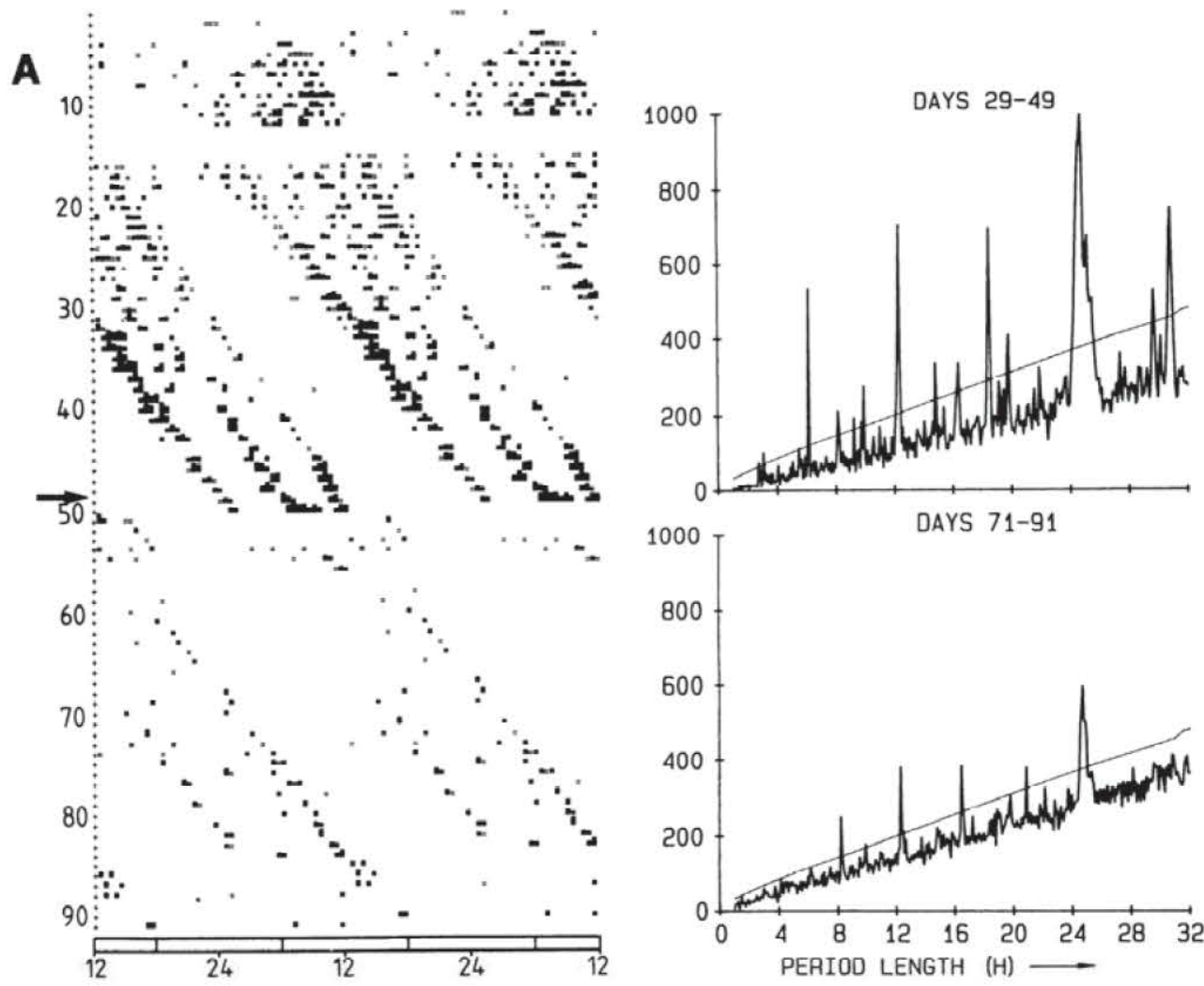

\section{LEW ণొ 5}
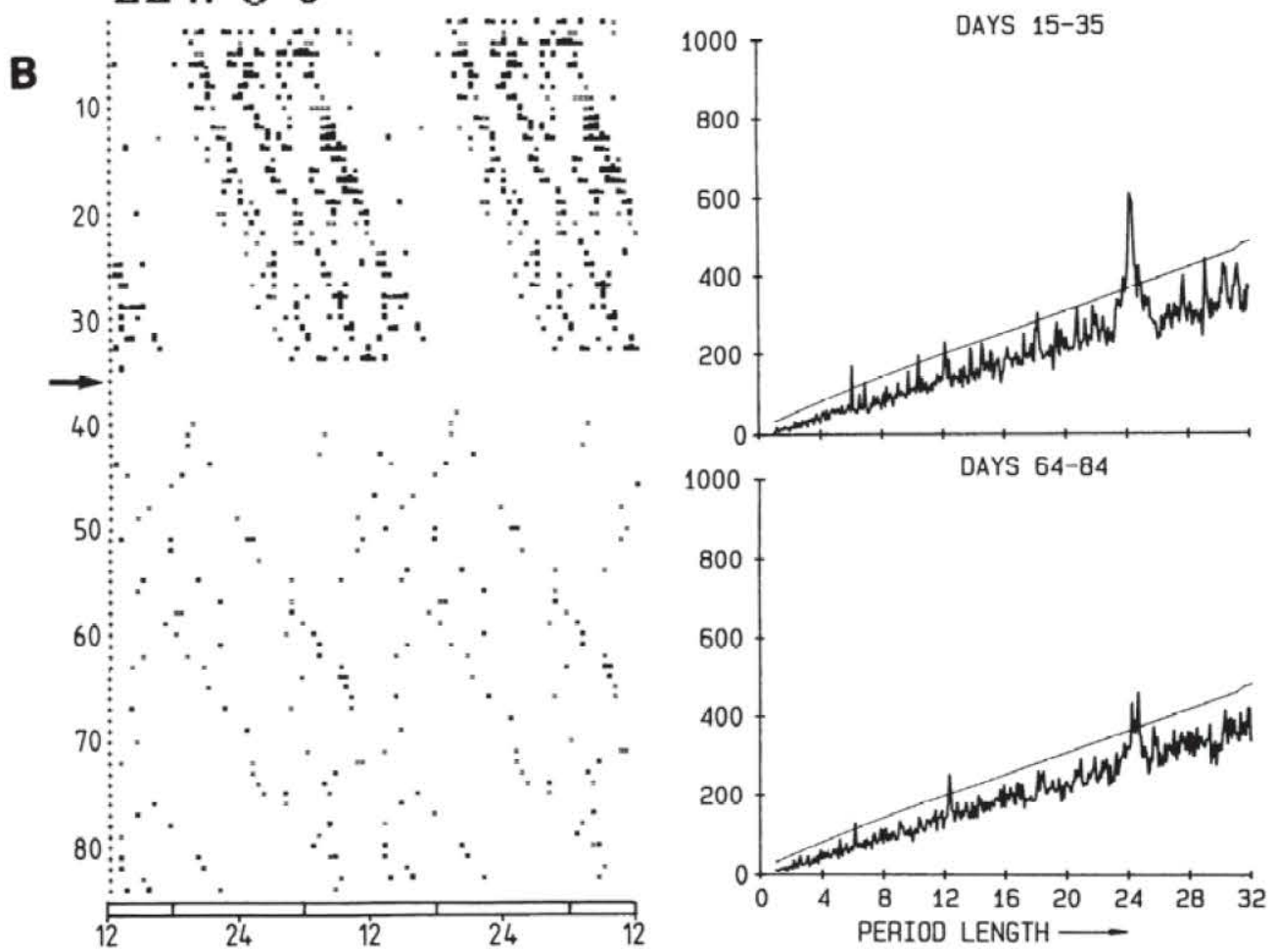

FIG. 5. Double-plotted wheel-running activity records of $6 \mathrm{LEW} / \mathrm{Ztm}$ rats with partial SCN lesions; $L E W{ }^{\star} 29$ with $85 \%$ damage of the SCN $(A), L E W \delta 5$ with $50 \%$ damage $(B), L E W \$ 11$ with $30 \%$ damage $(C), L E W \delta 3$ with $20 \%$ damage $(D), L E W \$ 19$ with $15 \%$ damage $(E)$, and $L E W \$ 18$ with damage only caudal to $\operatorname{SCN}(F)$. Each record is accompanied by 2 periodograms representing an interval of 21 days before and after surgery. For further explanation see Fig. 2.

reduction in the amplitude of the circadian spectral estimates. The spectral analysis also revealed that the decrease in the amplitudes of ultradian spectral estimates was statistically significant $(P<0.05)$ only in the male animals.
Relationship between ultradian and circadian rhythms. Periodogram analyses of all records showed that the ultradian periods were always submultiples of the circadian period. For animals entrained to a light-dark cycle, it is not surprising to find circadian rhythms of exactly 


\section{LEW $q 11$}
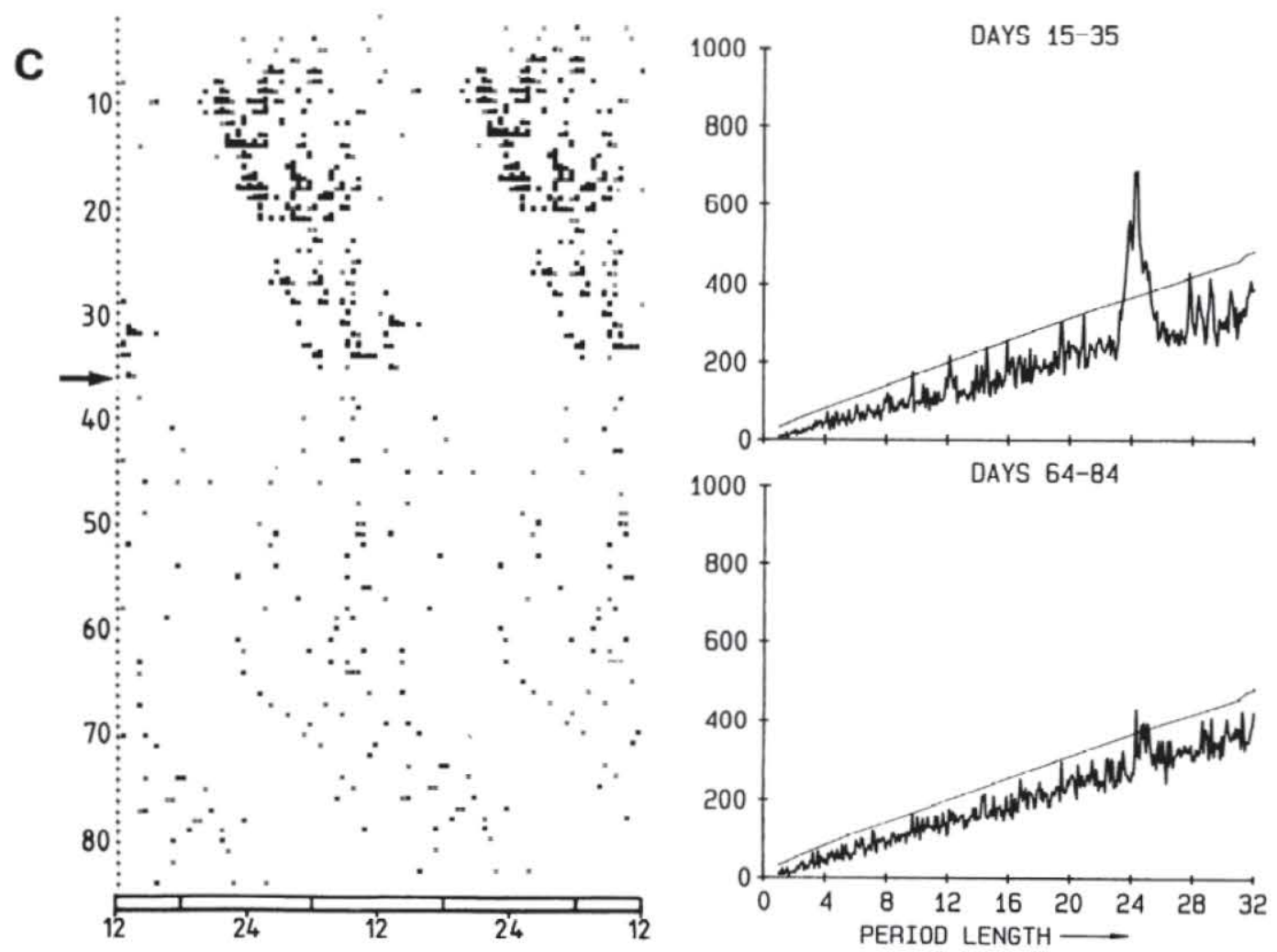

FIG. 5. Continued.

\section{LEW ơ 3}
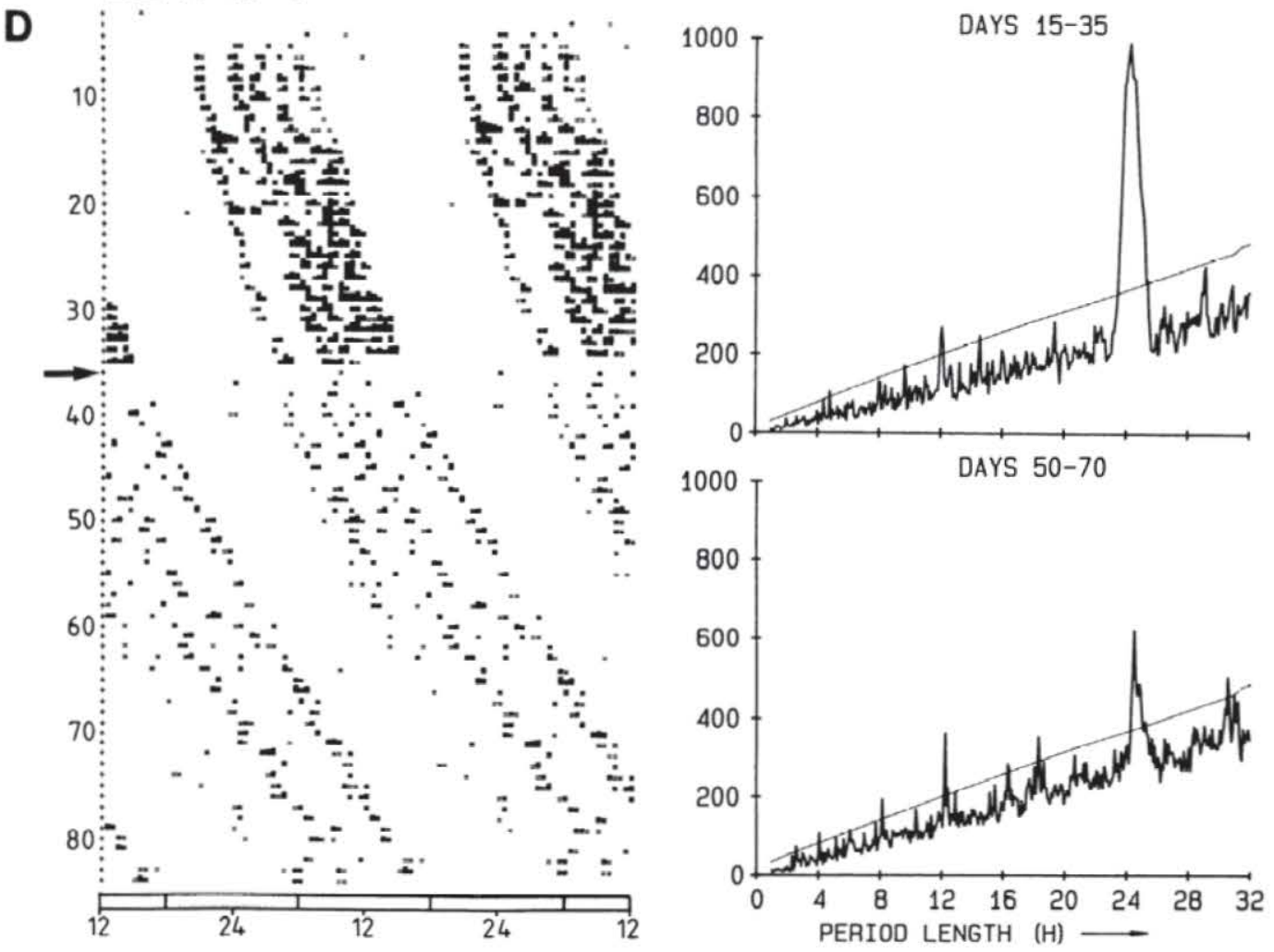

$24.0 \mathrm{~h}$ and ultradian peaks at 4 and $4.8 \mathrm{~h}$, but this relationship was also observed in the free-running animals. For example, periodogram analysis of $L E W \delta 29$ showed ultradian peaks at $6.17,4.95$, and $4.12 \mathrm{~h}$, which

are all submultiples of the circadian period $(24.67 \mathrm{~h})$ of this animal. Three particularly interesting cases were seen after partial SCN lesions. Periodogram analysis of the record of $L E W+19$ showed two different circadian 


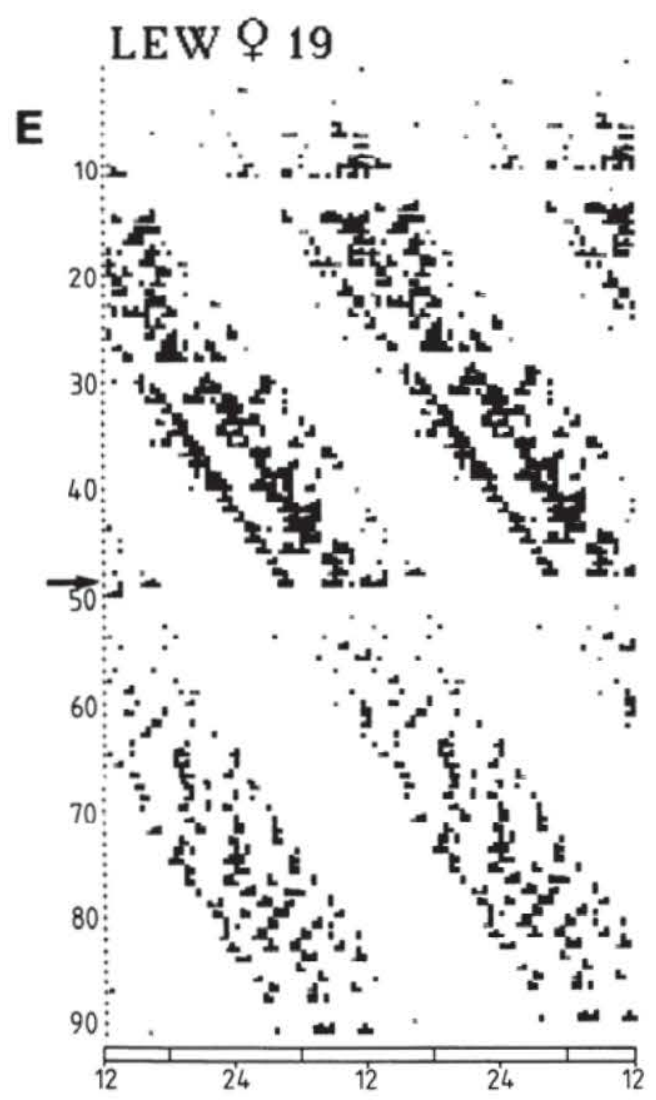

\section{LEW 우 18}

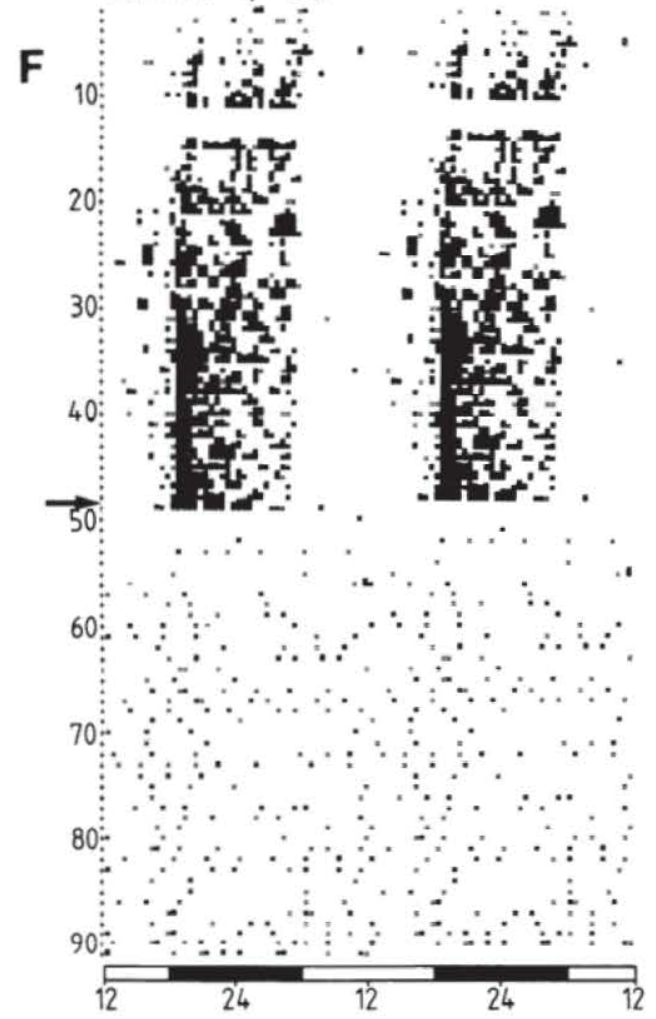

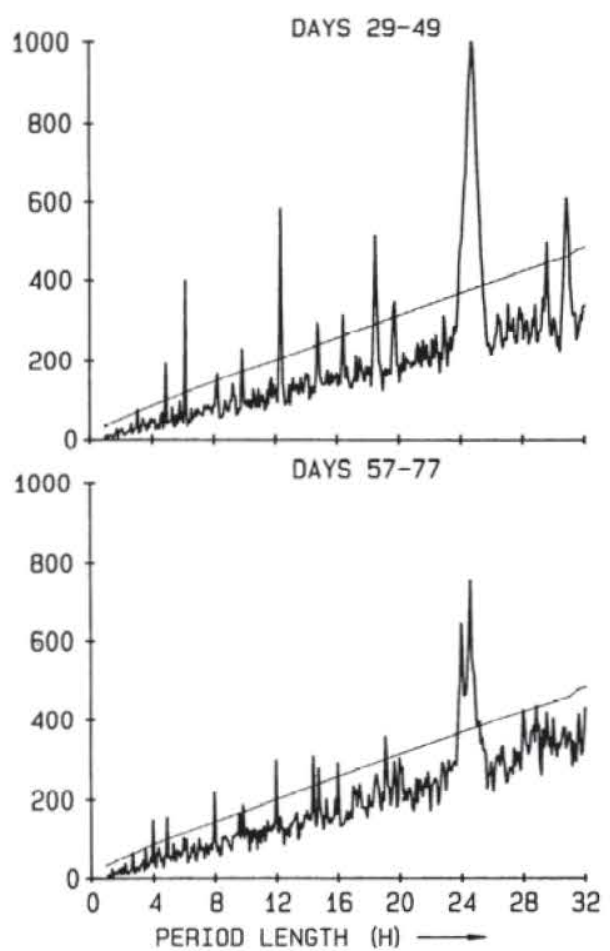

FIG. 5. Continued.

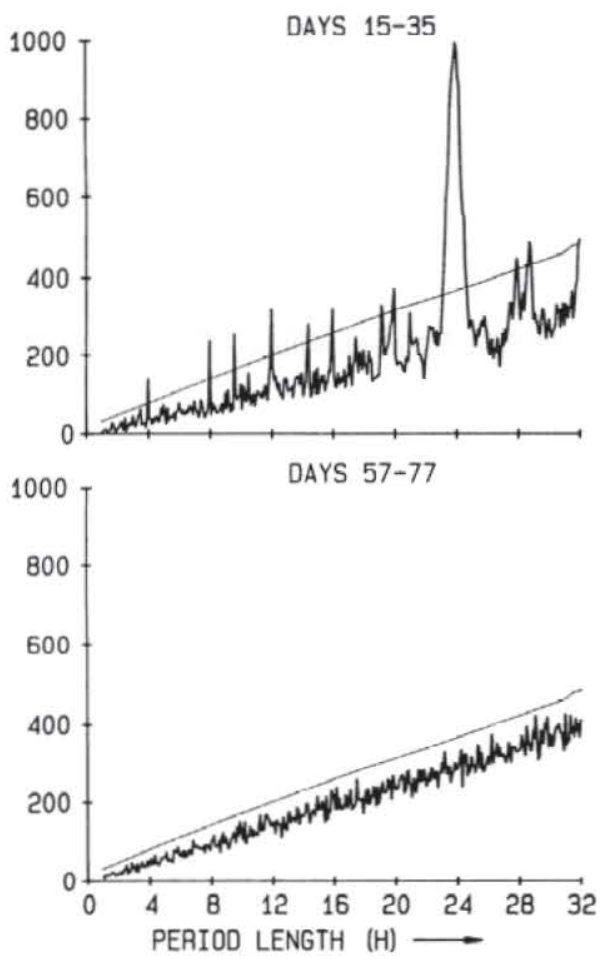

components for days 57-77, which were both associated with different ultradian submultiples (24.58 with $4.92 \mathrm{~h}$; 24.0 with $4.0 \mathrm{~h}$ ). $L E W \delta 5$ showed three circadian components, one of which was associated with an ultradian submultiple of $6.17 \mathrm{~h}$. LEW $\$ 11$ showed four different circadian components after the lesion without any ultradian submultiples.

Another indication of the close relationship between 
circadian and ultradian rhythms in the activity pattern of LEW/Ztm rats was the high correlation between the amplitudes of circadian and ultradian spectral estimates (Fig. 6). For this analysis, the amplitude values of the ultradian spectral estimates (i.e., 4.8- and 4-h values) were averaged and correlated with the amplitude values for the 24-h spectral estimates. Four data points from each animal, one for each time interval analyzed, were subject to linear regression analysis. Correlation of ultradian and circadian amplitudes was significantly different in males and in females, resulting in a regression line with a lesser slope for females $(m=0.19)$ than for males $(m=0.52)$. These results confirmed previous observations of sex differences in the expression of ultradian activity components in this strain (28).

\section{DISCUSSION}

Our findings in LEW/Ztm rats do not support the hypothesis that the trimodal modulation of the circadian wheel-running rhythm is caused by functionally and anatomically distinct ultradian oscillators. Instead the trimodal activity pattern appears to be generated by the circadian pacemaker system within the SCN region and may be the manifestation of a unique phase coupling between multiple circadian oscillators. This hypothesis is further supported by the fact that ultradian periods detected by periodogram analysis were always submultiples of the circadian period and by the high correlation between the amplitudes of circadian and ultradian spectral estimates in intact and partially $\mathrm{SCN}$-lesioned animals.

Whereas sham lesions had little or no effect on the activity pattern of wheel-running behavior in LEW/Ztm rats, complete SCN lesions resulted in the abolition of ultradian and circadian activity components in both male and female LEW/Ztm rats and in blinded free-running rats as well as in animals maintained under 12:12 LD conditions. Under both free-running or entrained conditions, circadian and ultradian activity components were still present in rats with partial SCN lesions. The amplitude of both ultradian and circadian spectral esti-

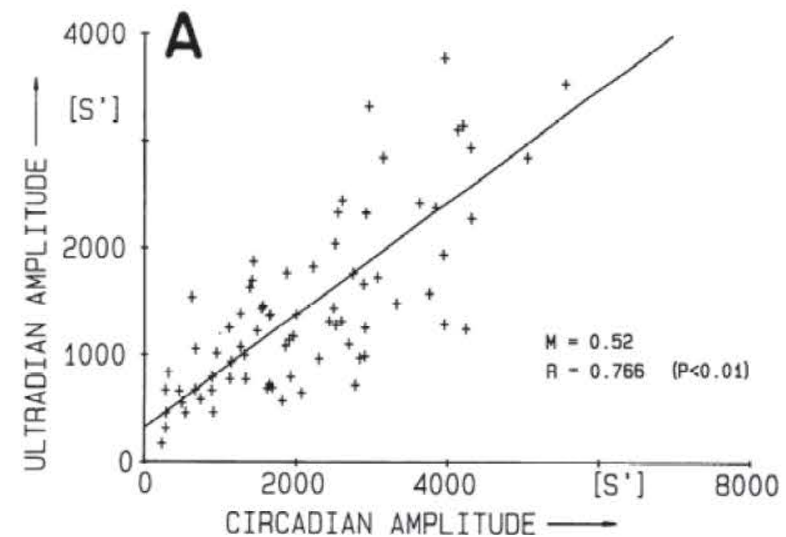

mates depended on the amount of SCN destroyed, as illustrated by the examples shown in Fig. 5. In animals $L E W \widehat{3} 3$ (Fig. $5 D$ ) and 119 (Fig. $5 E$ ), in which only 15$20 \%$ of the SCN was damaged, the trimodal activity patterns were similar to those observed before surgery, whereas in animals with larger lesions of the SCN only weak activity patterns were observed. For example, in $L E W \$ 29$ (Fig. $5 A$ ), only one of the three formerly visible activity bouts was present during days 58-68. A second component appeared a few days later.

The results of this study contrast with previous findings that indicated that ultradian components persist after SCN lesions in hamsters (21) and rats. Eastman et al. (9) reported ultradian variations in body temperature and sleep-wake recordings of rats, although they did not use periodogram analysis to verify period or amplitude. Other investigators $(12,16,27)$ described ultradian components in overall locomotor activity rhythms of SCNlesioned rats, but a statistical analysis of their data by periodogram and least-squares spectral analysis also revealed significant circadian peaks in most of the animals. According to Honma and Honma (12), this was probably due to a masking effect of light, since all the studies were done under light-dark entrainment. However, even under entrained conditions animals with complete $\mathrm{SCN}$ lesions in the present study failed to show any rhythmicity. $L E W$ 16 (Fig. 4A), for example, showed a totally arrhythmic activity pattern. In this animal, the visual system seemed to be intact because the lesion was very small and, specifically, there was no obvious damage to the optic tract or chiasm.

There are several differences between the present study and the investigations of Honma and co-workers $(12-14,16,27)$ that might account for the different results. One major difference is the method used for locomotor activity measurement. Whereas the present study investigated wheel-running activity, the studies of Honma and co-workers $(12-14,16,27)$ have been performed with an Animex system, an instrument that measures overall activity inside a regular animal cage and does not allow discrimination between different kinds of locomotor and feeding-related activities. The

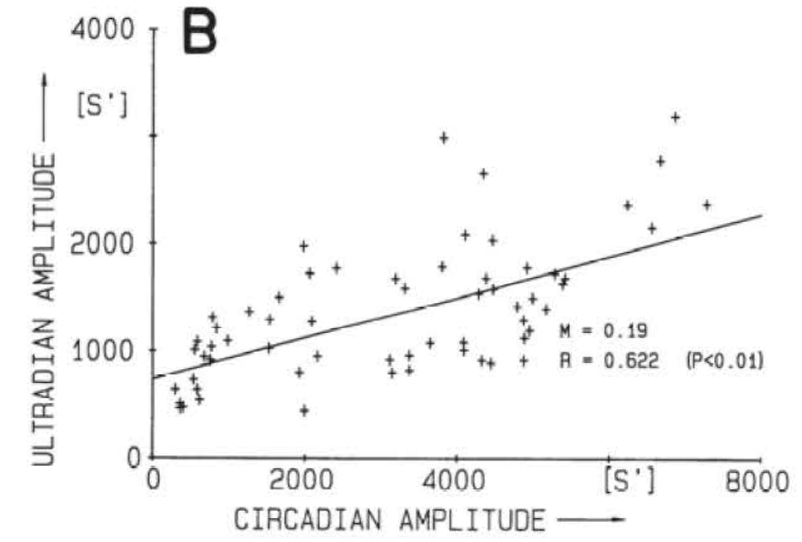

FIG. 6. Correlation between amplitudes of ultradian and circadian spectral estimates for male $(A)(n=76 ; m=$ $0.52 \pm 0.05 ; r=0.766, P<0.01)$ and female LEW/Ztm rats $(B)(n=64 ; m=0.19 \pm 0.03 ; r=0.622, P<0.01)$. For each animal 4 data points were plotted representing ultradian and circadian amplitudes given by power spectra of 4 different time intervals (compare with Fig. 3). Ultradian amplitude values were calculated as means of 4- and 4.8-h spectral estimates. 
effect of these different methods becomes quite obvious when the wheel-running activity patterns obtained in this study are compared with the overall activity patterns of the LEW/Ztm rats, which were found in previous studies with an Animex-like system (6). Measurements with the Animex-like system showed trimodal activity patterns only in male animals of the LEW/Ztm strain (28), whereas in this study ultradian activity components were found in both male and female rats. The runningwheel recordings actually showed an estrus-correlated modulation of the activity pattern in female LEW/Ztm rats, which is described in detail elsewhere (30). It seems that the running wheels provide a less noisy and more "filtered" activity recording than the Animex system because they measure a selected portion of the whole behavioral repertoire. However, previous investigations in LEW/Ztm rats have shown that a trimodal pattern is also expressed in other physiological parameters, e.g., body temperature and heart rate (5), food intake, and $\mathrm{O}_{2}$ consumption. The trimodal pattern, therefore, seems to be a general feature of the temporal organization of behavior in this inbred strain, which is expressed in wheel-running activity in a very precise fashion.

The second difference between this study and previous investigations is related to the period of the ultradian components. Ultradian periods found by Honma and coworkers $(12-14,16,27)$ in SCN-lesioned and SCN-isolated rats had means of 2.5 and $2.6 \mathrm{~h}$, respectively. These mean values are significantly different from the ultradian periods they reported for rats exposed to constant light, which were about twice as long (mean $=5.1 \mathrm{~h}$ ) (12). Because Honma and co-workers $(12-14,16,27)$ used an Animex system for activity recording, the remaining ultradian periods of $2.5 \mathrm{~h}$ may have been related to feeding or some other behavioral event that shows an ultradian rhythm.

Finally, there was a difference in the genetic background of the experimental animals. Although ultradian locomotor activity patterns have been reported for laboratory rats, they are not a general feature of the temporal organization of behavior as, for example, in voles (10). Descriptions of ultradian activity components in adult intact laboratory rats have been limited to the citation of a few unusual animals with trimodal or bimodal activity distributions that were "the major source of ultradian frequencies in the periodograms" (23). Ultradian components have been observed only under conditions such as continuous light $(1,13,14)$, after $\mathrm{SCN}$ lesions $(12,16$, 27 ), and during early developmental stages (15). The trimodal activity pattern of LEW/Ztm rats is, however, genetically fixed (6), displaying a recessive mode of inheritance (29). It seems likely that this strain carries a mutation in one or several of the gene loci that control the temporal organization of behavior.

Several properties of activity rhythms in vertebrates are best interpreted by a circadian timing system consisting of multiple oscillators and coordinated by both hierarchical and nonhierarchical (mutual) coupling relationships (20,21). Normally these oscillators interact to generate a coherent form of rhythmicity. However, certain conditions, such as continuous light, may cause desynchronization between these oscillators. For exam- ple, during long-term exposure to constant light, freerunning activity rhythms of hamsters frequently split into two distinct components, which may temporarily free-run at different periods, but usually stabilize in a $180^{\circ}$ phase relationship (25). Complete loss of rhythmicity or the occurrence of ultradian rhythms is often observed in rats exposed to constant light $(1,6,9,13,14)$.

One common feature of ultradian components detected in the activity patterns of intact or partial SCN-lesioned LEW/Ztm rats was the appearance of periods near the subharmonics of the 24-h rhythm. These submultiple peaks in the periodogram and spectral analysis are unlikely to be caused by nonstationary circadian rhythms for several reasons. 1) Periodogram analysis revealed the same ultradian periods in the activity pattern of LEW/ $\mathrm{Ztm}$ rats with no circadian rhythm because of long-term exposure to continuous light (6). 2) Tests of the periodogram and spectral analysis with artificial data (28) have shown that secondary peaks caused by nonstationary circadian rhythms appear in a different frequency range $(12,8$, and $6 \mathrm{~h})$ and show a different pattern. Furthermore, these tests have shown that the periodogram is capable of detecting nonharmonic ultradian rhythms of 5 or $7 \mathrm{~h}$, for example, in the presence of a circadian rhythm.

Further support for the hypothesis that the trimodal activity pattern in LEW/Ztm rats represents the interaction of multiple circadian oscillators is provided by the finding that the ultradian modulation is related to the hormonal status of adult animals. Estradiol released from long-term subcutaneous implants prevents the expression of ultradian activity patterns in male and female LEW/Ztm rats (28). Furthermore, we found an estruscorrelated modulation of the activity pattern in intact females of this strain, indicating a high correlation between the presence and absence of ultradian components and low and high estrogen levels, respectively (30). These results indicate that estrogen can change the phase relationship between the circadian oscillators that generate the trimodal activity pattern. Additional data have been presented that support the hypothesis that estradiol can strengthen the coupling between constituent oscillators. Morin (18) has demonstrated that administration of estradiol reduces splitting as well as other forms of abnormal rhythmicity in the activity rhythm of ovariectomized female hamsters exposed to constant light. These results indicate a functional similarity between splitting in hamsters and the trimodal activity pattern of LEW/Ztm rats.

In conclusion, the results of the present study have shown that complete SCN lesions abolish both the circadian rhythm of wheel-running behavior and the trimodal ultradian modulation of that activity. No evidence was found that ultradian activity rhythms persist after complete SCN lesions, as has been shown for the common vole (10). We cannot completely rule out the possibility of an ultradian pacemaker system within the $\mathrm{SCN}$, but it seems more likely that the trimodal activity pattern of the LEW/Ztm strain reflects the activity of multiple circadian oscillators. Caution is warranted not to generalize these results to other ultradian rhythms in rats as, for example, ultradian rhythms of hormone re- 
lease $(17,26)$. At present, there is no doubt that some ultradian rhythms are true periodic phenomena. However, along with the diversity of biological functions exhibiting ultradian rhythms (3), there is also a potentially wide variation in the processes generating such rhythms. Further examination of the LEW/Ztm strain should be useful for the development of a better understanding of the origin and function of ultradian activity components that have a clear association with the multioscillatory circadian system.

This research was supported by a grant from Deutsche Forschungsgemeinschaft to F. Wollnik (II 02-Wo 354/2-1) and by National Institute of Child Health and Human Development Grant HD-09885 to F. W. Turek.

Address for reprint requests: F. Wollnik, Universität Konstanz, Fakultät für Biologie, Postfach 5560, 7750 Konstanz 1, FRG.

Received 7 March 1988; accepted in final form 12 December 1988.

\section{REFERENCES}

1. Albers, H. E., A. A. Gerall, And J. F. Axelson. Circadian rhythm dissociation in the rat: effects of long-term constant illumination. Neurosci. Lett. 25: 89-94, 1981.

2. Ascноғғ, J. Spontane lokomotorische Aktivität. In: Handbuch der Zoologie, edited by J. G. Helmcke, H. von Lengerken, and D. Starck. Berlin: de Gruyter, 1962, vol. 8, p. 1-76.

3. Aschoff, J., AND M. P. GERKEMA. On diversity and uniformity of ultradian rhythms. In: Ultradian Rhythms in Physiology and Behavior, edited by H. Schulz and P. Lavie. New York: SpringerVerlag, 1985, p. 321-334.

4. BÜTTNER, D. Influence of strain specific patterns of locomotor activity on the daily pattern, minimal, mean and maximal oxygen consumption in the rat. Z. Versuchstierkd. 29: 121-128, 1987.

5. BUTTTNER, D., AND F. WollNIK. Spontaneous short-term fluctuations in the daily pattern of heart rate, body temperature, and locomotor activity in the laboratory rat. Lab. Anim. 16: 319-326, 1982.

6. BUtTNer, D., AND F. Wollnik. Strain-differentiated circadian and ultradian rhythms in locomotor activity of the laboratory rat. Behav. Genet. 14: 137-152, 1984.

7. Cheung, P. W., AND C. E. McCoRmack. Splitting of the locomotor activity rhythm in rats by exposure to continuous light. Am. J. Physiol. 244 (Regulatory Integrative Comp. Physiol. 13): R573R576, 1983.

8. DAAN, S., AND J. Aschoff. Short-term rhythms in activity. In: Handbook of Behavioral Neurobiology: Biological Rhythms, edited by J. Aschoff. New York: Plenum, 1981, vol. 4, p. 491-522.

9. Eastman, C. I., R. E. Mistlberger, and A. Rechtschaffen. Suprachiasmatic nuclei lesions eliminate circadian temperature and sleep rhythms in the rat. Physiol. Behav. 32: 357-368, 1984.

10. GERKEMA, M. P., AND S. DAAN. Ultradian rhythms in behavior: the case of the common vole (Microtus arvalis). In: Ultradian Rhythms in Physiology and Behavior, edited by H. Schulz and P. Lavie. New York: Springer-Verlag, 1985, p. 11-31.

11. Halberg, F., AND H. PANOFSKY. I. Thermo-variance spectra: methods and clinical illustrations. Exp. Med. Surg. 19: 285-321, 1961.

12. Honma, K. I., AND S. Honma. Ultradian rhythms in locomotor activity, deep body temperature and plasma corticosterone levels in rats: two different origins? In: Ultradian Rhythms in Physiology and Behavior, edited by $\mathrm{H}$. Schulz and P. Lavie. New York: Springer-Verlag, 1985, p. 77-94.

13. HoNMA, K. I., AND T. HIROSHIGE. Internal synchronization among several circadian rhythms in rats under constant light. $A m . J$. Physiol. 235 (Regulatory Integrative Comp. Physiol. 4): R243-R249, 1978.

14. Honma, K. I., AND T. Hiroshige. Endogenous ultradian rhythms in rats exposed to prolonged continuous light. Am. J. Physiol. 253 (Regulatory Integrative Comp. Physiol. 4): R250-R256, 1978.

15. HoNMA, S., AND K. I. Honma. Interaction between circadian and ultradian rhythms of spontaneous locomotor activity in rats during the early developmental period. In: Ultradian Rhythms in Physiology and Behavior, edited by H. Schulz and P. Lavie. New York: Springer-Verlag, 1985, p. 95-109.

16. Honma, S., K. I. Honma, and T. Hiroshige. Dissociation of circadian rhythms in rats with a hypothalamic island. Am. J. Physiol. 246 (Regulatory Integrative Comp. Physiol. 15): R949R954, 1984.

17. KNOBIL, E., AND J. HотснкISS. The circhoral gonadotropin releasing hormone (GNRH) pulse generator of the hypothalamus and its physiological significance. In: Ultradian Rhythms in Physiology and Behavior, edited by H. Schulz and P. Lavie. New York: Springer-Verlag, 1985, p. 32-40.

18. MoRIN, L. P. Effect of ovarian hormones on synchrony of hamster circadian rhythms. Physiol. Behav. 24: 741-749, 1980.

19. PANOFSKY, H., AND F. HALBERG. II. Thermo-variance spectra: simplified computational example and other methodology. Exp. Med. Surg. 19: 323-338, 1961.

20. Rosenwasser, A. M., AND N. T. AdLer. Structure and function in circadian timing systems: evidence for multiple coupled circadian oscillators. Neurosci. Behav. Rev. 10: 431-448, 1986.

21. RUSAK, B., AND I. ZUCKER. Neural regulation of circadian rhythms. Physiol. Rev. 59: 449-526, 1979.

22. Sokolove, P. G., AND W. N. Bushell. The chi square periodogram: its utility for analysis of circadian rhythms. J. Theor. Biol. 72: 131-160, 1978.

23. StePhan, F. K. Circadian rhythms in the rat: constant darkness, entrainment to $\mathrm{T}$ cycles and to skeleton photoperiods. Physiol. Behav. 30: 451-462, 1983.

24. Turek, F. W. Circadian neural rhythms in mammals. Annu. Rev. Physiol. 47: 49-64, 1985.

25. Turek, F. W., D. J. Earnest, and J. M. Swann. Splitting of the circadian rhythm of activity in hamsters. In: Vertebrate Circadian Systems: Structure and Physiology, edited by J. Aschoff, S. Daan, and G. A. Groos. New York: Springer-Verlag, 1982, p. 203-214.

26. VAN CAUTER, E., AND E. HoNINCKX. Pulsatility of pituitary hormones. In: Ultradian Rhythms in Physiology and Behavior, edited by H. Schulz and P. Lavie. New York: Springer-Verlag, 1985, p. 41-60.

27. Watanabe, K., and T. Hiroshige. Phase relations between episodic fluctuations of spontaneous locomotor activity and plasma corticosterone in rats with suprachiasmatic nuclei lesions. Neuroendocrinology 33: 52-59, 1981.

28. WOLLNIK, F., AND K. D. DöHLER. Effects of adult and perinatal hormonal environment on ultradian and circadian rhythms in locomotor activity of laboratory LEW/Ztm rats. Physiol. Behav. 38: 229-240, 1986.

29. WOLLNIK, F., K. GÄRTNER, AND D. BÜTTNER. Genetic analysis of ultradian and circadian locomotor activity rhythms in LEW/Ztm and ACI/Ztm rats. Behav. Genet. 17: 167-178, 1987.

30. WOLLNIK, F., AND F. W. TUREK. Estrous correlated modulations of circadian and ultradian wheel-running activity rhythms in LEW/Ztm rats. Physiol. Behav. 43: 389-396, 1988. 